\title{
CT and MRI Imaging Diagnosis of Epidural Idiopathic Sterile Pyogranulomatous Inflammation in a Dog Spinal Canal
}

\author{
Daiki MURATA ${ }^{1)}$, Naoki MIURA ${ }^{1,2)^{*}}$, Tomoko IWANAGA ${ }^{1)}$, Satoshi TOKUNAGA ${ }^{3)}$, Noriaki MIYOSHI ${ }^{4)}$, \\ Yoshiaki KITANO ${ }^{5}$, Yasuyuki MOMOI ${ }^{2}$ and Makoto FUJIKI $^{3)}$ \\ 1) Veterinary Teaching Hospital, Joint Faculty of Veterinary Medicine, Kagoshima University, 1-21-24 Korimoto, Kagoshima \\ 890-0065, Japan \\ ${ }^{2)}$ Laboratory of Diagnostic Imaging, Joint Faculty of Veterinary Medicine, Kagoshima University, 1-21-24 Korimoto, Kagoshima \\ 890-0065, Japan \\ ${ }^{3)}$ Laboratory of Surgery, Joint Faculty of Veterinary Medicine, Kagoshima University, 1-21-24 Korimoto, Kagoshima 890-0065, \\ Japan \\ 4)Laboratory of Pathology, Joint Faculty of Veterinary Medicine, Kagoshima University, 1-21-24 Korimoto, Kagoshima 890-0065, \\ Japan \\ ${ }^{5)}$ Kitano Animal Hospital, 299 Heiwa-cyo, Izumi 899-0217, Japan
}

(Received 23 July 2011/Accepted 25 January 2012/Published online in J-STAGE 8 February 2012)

ABSTRACT. A 12-year-old neutered male shih tzu developed progressive pelvic limb paraparesis. Computed tomography showed a radiolucent mass lesion in the spinal canal at the left side of the 11th thoracic vertebra. The mass was not enhanced by intravenous contrast medium injection. It was hyperintense on both T1- and T2-weighted magnetic resonance images. The signal intensity of the mass was decreased with a fat suppression technique, indicating a fatty origin. After removal of the mass via T11-T12 hemilaminectomy, chronic panniculitis was confirmed by histopathological examination. This case demonstrates the utility of computed tomography and magnetic resonance imaging for the diagnosis of spinal canal pyogranulomatous inflammation.

KEY WORDS: computed tomography, magnetic resonance image, spinal canal pyogranulomatous inflammation.

doi: 10.1292/jvms.11-0348; J. Vet. Med. Sci. 74(7): 913-915, 2012

Epidural idiopathic sterile pyogranulomatous inflammation is inflammation of the panniculus adiposus. It exists as a single mass or multiple masses in the subcutaneous tissue [7]. To our knowledge, only one report has described epidural idiopathic sterile pyogranulomatous inflammation in the spinal canal causing neurological deficits [1]. A mildly radiolucent epidural mass impinged upon the spinal cord on myelographic images [1]. However, computed tomography (CT) and magnetic resonance (MR) imaging has not been previously discussed. Epidural idiopathic sterile pyogranulomatous inflammation and infiltrative lipomas have very similar features on both CT and MR images, and both could be consistent with a fatty mass in the spinal canal. The usefulness of MR imaging for the diagnosis of infiltrative lipomas has been reported [5]. Differences between images of the two diseases have not been compared. Complete removal of epidural idiopathic sterile pyogranulomatous inflammation in the spinal canal leads to a good prognosis; in contrast, it is difficult to totally remove infiltrative lipomas, which results in a high rate of recrudescence. Therefore, it is important to differentiate the two diseases for appropriate

\footnotetext{
*Correspondence to: Miura, N., Veterinary Teaching Hospital and Laboratory of Diagnostic Imaging, Joint Faculty of Veterinary Medicine, Kagoshima University, 1-21-24 Korimoto, Kagoshima 890-0065, Japan.

e-mail: k9236024@kadai.jp

(C) 2012 The Japanese Society of Veterinary Science
}

treatment strategy planning. We herein describe the utility of CT and MR imaging for diagnosis of pyogranulomatous inflammation in the spinal canal, the treatment strategy, and the prognosis.

A 12-year-old neutered male shih tzu was examined for pelvic limb paraparesis that developed over a period of 9 weeks. There was generalized weakness and lethargy. The dog was unable to stand on its pelvic limbs without assistance. The dog showed upper motor neuron signs, and neurological abnormalities included conscious proprioception and superficial pain perception deficits in the pelvic limbs, deep pain perception deficits in the left hind leg (which was more affected than the right), and cutaneous trunci reflex deficits on the left side caudally from around the 11th thoracic (T11) vertebra. Moreover, dysuria was observed. On 4-row helical CT images (Asteion TSX-021B; TOSHIBA $®$, Otahara, Japan), there was a radiolucent lesion within the spinal canal at T11 that was located ventrally to the spinal cord, lateralized to the left, and had a CT value of $-38 \mathrm{HU}$ (Fig. 1). The T11 vertebral bone had no abnormal signs. The lesion was not enhanced by intravenous contrast medium injection. No signs of intervertebral disc space narrowing or intervertebral disc displacement were noted. On 0.4-Tesla MR images (Aperto/Aperto Inspire; HITACHI ${ }^{\circledR}$, Tokyo, Japan), a high-signal-intensity lesion was observed on T1weighted (Fig. 2A) and T2-weighted (Fig. 2B) images. The lesion was well circumscribed, and the length of the lesion was equivalent to one vertebral body. The spinal cord was 


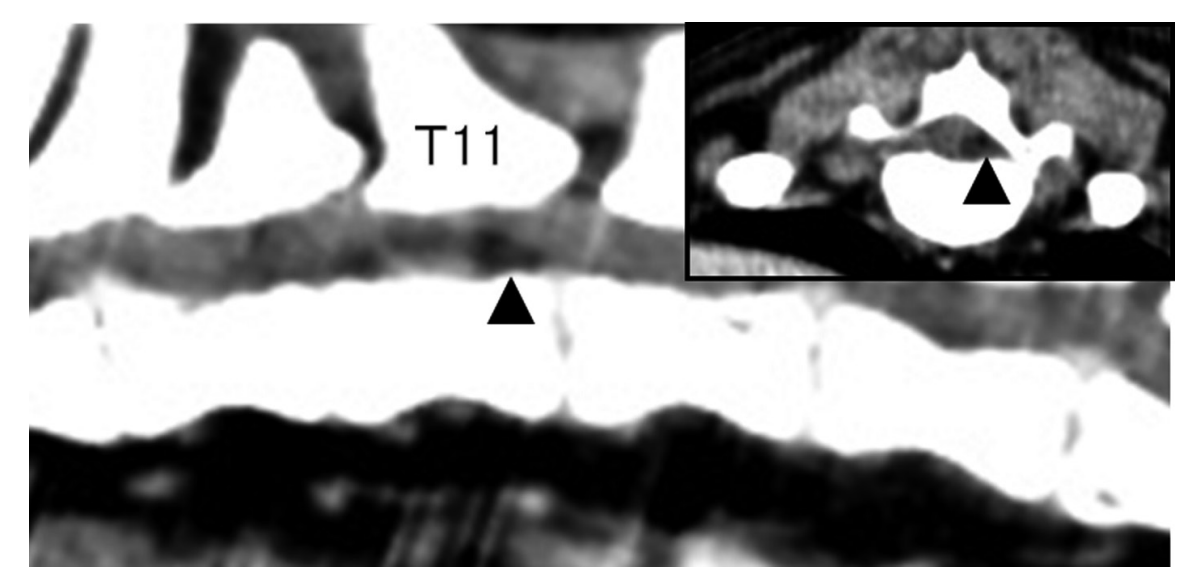

Fig. 1. Computed tomography images

On computed tomography (CT) imaging, there was a radiolucent lesion (arrowhead) within the spinal canal at 11th thoracic vertebra that was located ventral to spinal cord and lateralized to the left (window). The $\mathrm{CT}$ value of the lesion was $-38 \mathrm{HU}$.

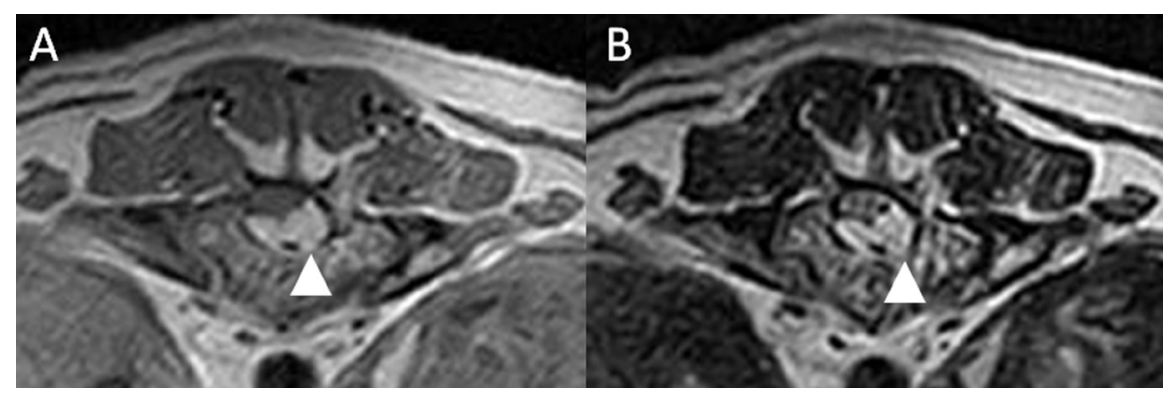

Fig. 2. Magnetic resonance images (transverse)

On magnetic resonance images, there was a high-signal lesion (arrowhead) on T1- (A) and T2-weighted (B) images. The lesion was well circumscribed. The spinal cord was compressed and displaced to the right and dorsally by the mass. No abnormal findings were noted in the muscle surrounding the T11 vertebra.

compressed and displaced dorsally by the mass. No abnormal findings were noted in the muscle adjacent to the T11 vertebra. However, the edges of the high-signal mass lesion on the T1- and T2-weighted images demonstrated a low signal via a fat suppression technique, while the central lesion of the mass still produced a high signal (Fig. 3). Hemilaminectomy was performed on the left anterior articular process of T11 and the left retroarticular process of T12 under general anesthesia (propofol induction followed by oxygen-isoflurane inhalation maintenance). A mass entered the vertebral canal and impinged upon the spinal cord. The mass was similar in appearance to fat and well circumscribed. The mass was extracted from the vertebral canal, and adhesion to the spinal dura mater was not observed. Histopathological findings were benign, well-differentiated adipose tissue; osteoid formation; and infiltration of lymphocytes and plasma cells, which were consistent with pyogranulomatous inflammation. Thus, the lesion was diagnosed as chronic panniculitis. Furthermore, the central lesion of the mass comprised hard, osteoid tissue. Five months after surgery, the dog remained fully ambulatory with no neurological deficits. In this animal, epidural idiopathic sterile pyogranulomatous inflammation was identified on the T11 portion of the spinal cord, causing progressive pelvic limb paresis.

To our knowledge, this is the first report to describe CT and MR imaging of an epidural idiopathic sterile pyogranulomatous inflammatory mass in the spinal canal. There was a radiolucent lesion $(-38 \mathrm{HU})$ within the spinal canal on CT images, and the lesion was not enhanced by contrast medium. The vertebral bone had no abnormal findings (Fig. 1). The adipose tissue was easily distinguishable by CT imaging, because of its unique quantification of X-ray resorption; fat tissue showed negative Hounsfield unit values (less than $-20 \mathrm{HU})$ [7]. In addition, CT provides the best definition of bone structure. There was no obvious destruction of the vertebral body, so the fatty mass confined to the spinal canal. On MR images, there was a hyperintense mass extending into the left aspect of the T11 vertebral canal on both T1- and T2 weighted images. Because MR imaging provides the best definition of soft tissue, the muscle around T11 showed no changes. Moreover, because fat suppression techniques are useful in ascertaining the fatty characteristics of a mass [2, 4-6], the mass lesion in the present case was exactly defined by a fat suppression technique. Differential diagnoses for an MR image like that in the present case in which T1- and T2-weighted images showed hyperintense signals include 

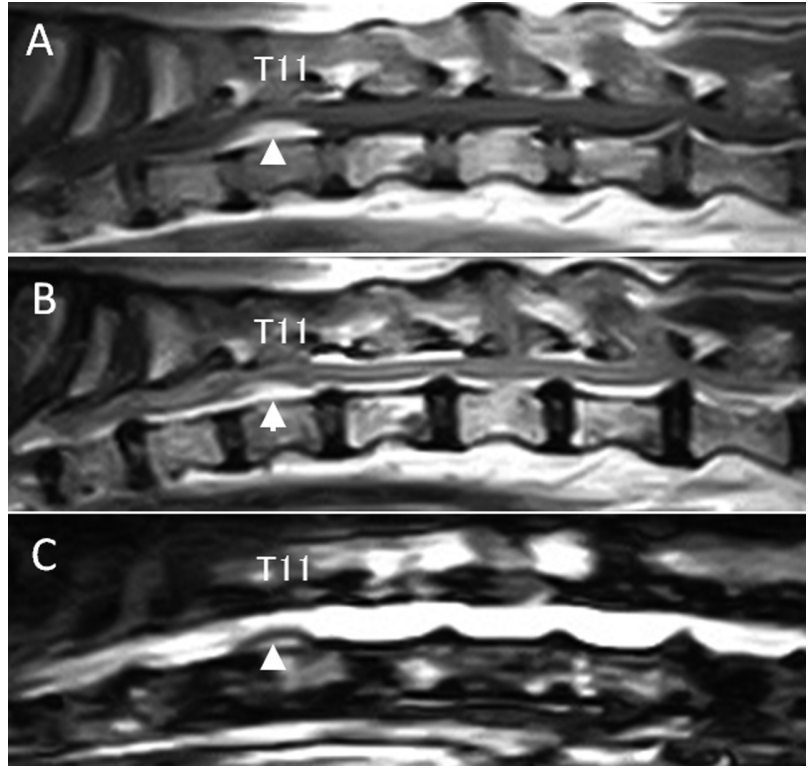

Fig. 3. Magnetic resonance images (sagittal)

On magnetic resonance images, a high-signal lesion was detected on T1-weighted (A) and T2-weighted (B) images. The length of the lesion was equivalent to one vertebral body (arrowhead). The spinal cord was compressed and displaced dorsally by the mass. The edges of the highsignal mass lesion on the T1- and T2-weighted images demonstrated a low signal via a fat suppression image, while the central lesion of the mass still produced a high signal (C).

hemorrhage, hematoma, and fatty tissue $[1,3-6,8]$. Of these, a fat suppression image may be useful to distinguish the involvement of adipose tissue amongst other possible etiologies [2, 4-6]. In addition, CT was also a powerful tool in this case in determination of a fatty mass, because the only substance other than adipose tissue that demonstrates negative Hounsfield unit values is air $[1,4]$. The difference between epidural idiopathic sterile pyogranulomatous inflammation and infiltrative lipomas may be the origin of the fatty tissue. The adipose tissue in infiltrative lipomas originally arises in the muscle and then infiltrates the spinal cavity, but pyogranulomatous inflammation is confined within the spinal canal $[1,5]$. To thoroughly examine the fatty tissue, both $\mathrm{CT}$ and MR images are useful for distinct reasons. An MR image can clearly indicate the border between the fatty tissue and other soft tissue components $[2,5,6]$. A CT image, however, is also able to detect fatty infiltration in the muscle area around the spinal cord as negative Hounsfield unit values $[4,6]$. Moreover, CT imaging is useful to detect destruction of the vertebral body, which is a common feature of infiltrative lipomas [3]. Another important issue in the differential diagnosis of the 2 diseases is planning the surgical strategy. In the present case and in previous report, the dog completely recovered by removal of the pyogranulomatous inflammation [1]. Aggressive surgical treatment should be recommended for dogs affected with pyogranulomatous inflammation. It is relatively easy for a surgeon to remove the mass, if it is confined to the vertebral canal, as in cases of pyogranulomatous inflammation [1]. Although the prognosis is good for dogs in whom infiltrative lipomas are completely removed, complete excision is difficult, because the mass originates from the muscle and infiltrates the spinal canal $[2,4,5]$. The images reported here demonstrate the utility of CT and MR imaging for diagnosis of pyogranulomatous inflammation in the spinal canal. Because pyogranulomatous inflammation is composed of adipose tissue, these masses are characterized by negative Hounsfield units on CT images; typically, adipose tissue shows Hounsfield units of less than $-20 \mathrm{HU}$. The mass was also observed to have a high-signal intensity on both T1- and T2-weighted images as well as within the center of the fat suppression image, although signal inhibition was evident along the lesion edges. Pyogranulomatous inflammation includes an inflamed reaction within the lesion, which is uncommon in lipomas. In the present case, the high-signal intensity of the center of the mass on fat suppression images was pathologically diagnosed as reactive osteoid, in which the signal intensity was similar to that of the vertebral bone cortex. Of particular importance is that CT and MR imaging are useful modalities for investigation of vertebral body destruction and soft tissue conditions, respectively.

ACKNOWLEDGMENTS. The authors would like to thank the veterinarians at Kitano Animal Hospital for supplying case information and Dr. Michael J. Mienaltowski for English instruction and assistance.

\section{REFERENCES}

1. Aikawa, T., Yoshigae, Y. and Kanazono, S. 2008. Epidural idiopathic sterile pyogranulomatous inflammation causing spinal cord compressive injury in five Miniature Dachshunds. Vet. Surg. 37: 594-601. [Medline] [CrossRef]

2. Kim, H. J., Chang, H. S., Choi, C. B., Song, Y. S., Kim, S. M., Lee, J. S. and Kim, H. Y. 2005. Infiltrative lipoma in cervical bones in a dog. J. Vet. Med. Sci. 67: 1043-1046. [Medline] [CrossRef]

3. Mateo, I., Lorenzo, V., Foradada, L. and Muñoz, A. 2011. Clinical, pathologic, and magnetic resonance imaging characteristics of canine disc extrusion accompanied by epidural hemorrhage or inflammation. Vet. Radiol. Ultrasound 52: 17-24. [Medline]

4. McEntee, M. C. and Thrall, D. E. 2001. Computed tomographic imaging of infiltrative lipoma in 22 dogs. Vet. Radiol. Ultrasound 42: 221-225. [Medline] [CrossRef]

5. Morgan, L. W., Toal, R., Siemering, G. and Gavin, P. 2007. Imaging diagnosis-infiltrative lipoma causing spinal cord compression in a dog. Vet. Radiol. Ultrasound 48: 35-37. [Medline] [CrossRef]

6. Pereira, J. M., Sirlin, C. B., Pinto, P. S. and Casaola, G. 2005. CT and MR imaging of extrahepatic fatty masses of the abdomen and pelvis: techniques, diagnosis, differential, diagnosis, and pitfalls. Radiographics 25: 69-85. [Medline] [CrossRef]

7. Scott, D. W., Miller, W. H. and Griffion, C. E. 2001. Miscellaneous skin disease. pp. 1136-1140 and 1156-1182. In: Miller and Kirk's Small Animal Dermatology 6th ed. (Scott, D. W., Miller, W. H. and Griffion, C. E. eds.), Saunders, Philadelphia.

8. Tidwell, A. S., Spech, A., Blaeser, L. and Kent, M. 2002. Magnetic resonance imaging features of extradural hematomas associated with intervertebral disc herniation in a dog. Vet. Radiol. Ultrasound 43: 319-324. [Medline] [CrossRef] 